Spectral and scattering theory, Plenum publishers,

New York, 1998 (ed. A.G.Ramm) pp 89-110.

\title{
SCATTERING BY OBSTACLES IN ACOUSTIC WAVEGUIDES
}

\author{
A.G. RAMM * \\ Department of Mathematics, Kansas State University, \\ Manhattan, Kansas 66506-2602, U.S.A. \\ G.N. MAKRAKIS ${ }^{\dagger}$ \\ Institute of Applied and Computational Mathematics, FO.R.T.H \\ P.O. Box 1527, 71 110, Heraklion, Crete, Greece \\ and \\ Department of Mathematics, University of Crete
}

\begin{abstract}
We study the scattering of acoustic waves by an obstacle embedded in a uniform waveguide with planar boundaries, which is a fundamental model in shallow ocean acoustics. Under certain geometric assumptions on the shape of the obstacle, we prove a Rellichtype uniqueness theorem in the case of soft obstacles. The solvability of the scattering problem is proved via boundary integral equations and the limiting absorption principle is established. An eigenfunction expansion in terms of the scattering solutions reveals the appropriate (partial) scattering amplitudes for the waveguide. We show that these scattering amplitudes for the propagating modes, at a fixed frequency, define uniquely the shape of a soft obstacle.
\end{abstract}

\section{Introduction}

In this paper we deal with some aspects of the scattering of acoustic waves by an obstacle embedded in a uniform waveguide with planar boundaries, which is a fundamental model for shallow-ocean acoustics ${ }^{1}$. This model has been already considered for acoustically soft obstacles (Dirichlet boundary condition) by Gilbert and $\mathrm{Xu}{ }^{5,6,7,28}$ who have also dealt with the case of a horizontally stratified ocean.

It is well known that the Dirichlet Laplacian has purely continuous spectrum for a large class of unbounded domains, including exterior of bounded domains, some classes of generalized (perturbed) conical domains, generalized cylinders and locally perturbed generalized cylinders (see, e.g., Refs. 4,10,16,25). The relation between

\footnotetext{
*E-mail: ramm@math.ksu.edu

†E-mail: makrakg@calderon.iacm.forth.gr
} 
the spectral properties of linear operators, in particular, the Schrödinger operators, and the behavior at large times of the solutions to exterior time-dependent problems was studied by Ramm ${ }^{10}$, and for the waveguide by Ramm and Werner ${ }^{24}$.

If the local perturbation violates certain geometrical restrictions (like the one in the Assumptions (A) or (B) in Section 2 below), it is possible to have an unbounded sequence of eigenvalues which are embedded in the essential spectrum (see Section 7 below, and Ref. 25 and the references therein). The same situation arises also in the case of the Neumann Laplacian. It has been shown that in certain two- dimensional water-wave and acoustic problems governed by the Helmholtz equation in strips (see, e.g., Refs. 2 and 3), trapped modes can exist in the case of hard obstacles. Therefore, the conditions which the obstacles must satisfy for the uniqueness of the solution to the scattering problem in waveguides to hold are not well understood. It is an open problem to clarify these assumptions. In particular, uniqueness theorem for scattering or boundary-value problem for a hard obstacle in the waveguide is presently not known and non-uniqueness results are given in Ref 2,3.

We present some uniqueness results for soft obstacles in a waveguide in Section 2. The first of our proof uses an idea of Rellich ${ }^{25}$ but is based on the new identity (2.4), and the second one is based on the Rellich's identity. A uniqueness theorem similar to our Theorem 2.1 for the case of soft obstacles has been given earlier by Morgenröther and Werner ${ }^{8}$, but our proof is different. The second uniqueness Theorem 2.1' is new.

In Section 3 we investigate the solvability of the boundary value problem for a soft obstacle using boundary integral equations. In Section 4 we prove the limiting absorption principle for the problem at hand, following Ramm ${ }^{16}$ (see also Ref. 10, Chapt. VII, Sec. 5). An appropriate definition of the scattering solutions is given in Section 5, and an eigenfunction expansion leading to a natural definition of the partial scattering amplitudes for the waveguide problem is also constructed there. In Section 6 the uniqueness of an inverse obstacle scattering problem at fixed frequency (that is, the assertion that the scattering data, namely partial scattering amplitudes for the propagating modes, uniquely define the shape of the obstacle) is proved following the reasoning in Ref. 10, pp. 85-86, and the results and ideas in Ref. 17. In section 7 an example of eigenvalues embedded in the continuous spectrum of the Dirichlet Laplacian in a locally perturbed and rotationally symmetric waveguide is given. This example is taken essentially from the paper by Witsch ${ }^{26}$.

In section 8 some recent papers by R.Kleinman, T.Angell and coauthors are discussed. In these papers a uniqueness theorem is claimed for a boundary value problem for hard obstacle in a waveguide. We point out a mistake in their proof. The uniqueness theorem, claimed by the mentioned authors, is wrong, and a counterexample can be found in Refs. 2,3. We also point out that the "new conditions" introduced in these recent papers in place of the radiation condition, are void: they are never satisfied. We follow Ref. 23 in Section 8.

We do not want to increase the volume of the paper, and that is why some results similar to the ones in Refs. 11-22 are not stated and discussed. We refer 
the reader to Refs. 15 and 17 for analysis of the scattering problems in the case of Lipschitz boundaries, to Refs. 11-13 and 17 for some methods of the proof of the uniqueness of the solution to inverse obstacle scattering problems, in particular for non-smooth obstacles, for instance, obstacles with Lipschitz (and even less regular) boundaries, to Ref. 21 for an analytical example of non-uniqueness of the solution to an inverse geophysical problem, and to Ref. 18 for a study of the dependence of the scattering amplitude on the boundary of the obstacle.

We consider the homogeneous waveguide, but the theory we develop is applicable without essential changes to the horizontally stratified waveguide. This is clear from the presentation (see also the closing paragraph at the end of section 3).

We will use the following notations. Let $L:=\left\{x=\left(\widehat{x}, x_{3}\right): \widehat{x} \in \mathbb{R}^{2}, 0 \leq\right.$ $\left.x_{3} \leq h\right\}$, where $\widehat{x}=\left(x_{1}, x_{2}\right)$ and $h$ is a positive constant, and $D$ be a bounded connected subdomain of $L$ with $C^{2}$ boundary $S$ having a unit normal $N$ pointing into $D$. We denote the complement of $D$ in $L$ by $D^{\prime}:=L \backslash D$, and let $D_{r}:=$ $\left\{x:|\widehat{x}| \leq r, x \in D^{\prime}\right\}, \partial D_{r}=S \cup \Gamma_{r} \cup \partial L_{r}, \Gamma_{r}:=\left\{x:|\widehat{x}|=r, x \in D^{\prime}\right\}$ and $\partial L_{r}:=\{x: x \in \partial L,|\widehat{x}| \leq r\}$. By $r_{0}>0$ we denote such a number, that the obstacle $D$ lies in the cylinder $\left\{x: x \in L,|\hat{x}|<r_{0}\right\}$. Let $L_{0}^{2}\left(D^{\prime}\right)$ stand for the set of $L^{2}\left(D^{\prime}\right)$-functions vanishing near infinity (but not necessarily near $S$ ), and $\Omega_{0}$ for the exterior in $L$ of the region $D_{r_{0}}$.

Scattering of acoustic waves in the layer $L$ is governed by the Helmholtz equation

$$
\Delta u+k^{2} u=0 \quad \text { in } \quad D^{\prime}, \quad k=\text { const }>0 .
$$

We assume that $u$ satisfies either the boundary conditions

$$
u(\widehat{x}, 0)=u(\widehat{x}, h)=0,
$$

or

$$
u(\widehat{x}, h)=\partial_{x_{3}} u(\widehat{x}, 0)=0,
$$

on the surfaces $x_{3}=0$ and $x_{3}=h$ of the layer $L$. We fully investigate the case $\left.u\right|_{S}=0$ (soft obstacle), and we comment on the case $\left.\partial_{N} u\right|_{S}=0$ (hard obstacle).

If the obstacle is absent the above problem can be solved by taking the Fourier transform of equation (1.1) with respect to the variable $\hat{x}$. This yields the eigenvalue problem

$$
\begin{gathered}
\phi^{\prime \prime}\left(x_{3}\right)+k^{2} \phi\left(x_{3}\right)=\lambda^{2} \phi\left(x_{3}\right), \\
\phi(0)=\phi(h)=0,
\end{gathered}
$$

or

$$
\phi(h)=\phi^{\prime}(0)=0,
$$

corresponding to the boundary conditions (1.2) or (1.3), respectively. Here

$$
\phi:=\frac{1}{2 \pi} \int_{\mathbb{R}^{2}} u\left(\hat{x}, x_{3}\right) \exp (i \boldsymbol{\lambda} \cdot \hat{x}) d \hat{x},
$$

and $\phi:=\phi\left(x_{3}, \lambda, k\right), \boldsymbol{\lambda}:=\left(\lambda_{1}, \lambda_{2}\right),|\boldsymbol{\lambda}|:=\lambda$. 
The problems (1.4), (1.5) (or (1.4), (1.6)) have an infinite set of simple eigenvalues

$$
\lambda \in \mathcal{K}:=\left\{\lambda_{m}=m \frac{\pi}{h}, \quad m=1,2, \ldots\right\}
$$

or

$$
\lambda \in \mathcal{K}^{\prime}:=\left\{\lambda_{m}=\left(m+\frac{1}{2}\right) \frac{\pi}{h}, \quad m=0,1,2, . .\right\},
$$

respectively. The corresponding eigenfunctions $\phi_{m}\left(x_{3}\right)$ form a complete orthonormal set in $L_{2}(0, h)$ if we also impose the normalization condition

$$
\int_{0}^{h}\left|\phi_{m}\right|^{2} d x_{3}=1
$$

where

$$
\phi_{m}\left(x_{3}\right)=\sqrt{\frac{2}{h}} \sin \left(m \pi x_{3} / h\right), \quad m=1,2, \ldots,
$$

if condition (1.5) holds, or

$$
\phi_{m}\left(x_{3}\right)=\sqrt{\frac{2}{h}} \cos \left(\left(m+\frac{1}{2}\right) \pi x_{3} / h\right), \quad m=0,1,2, \ldots,
$$

if condition (1.6) holds.

\section{The uniqueness theorem}

In the sequel we prove a uniqueness theorem for the scattering problem when the obstacle $D$ is soft. We assume in this paper, unless otherwise stated, that the obstacle satisfies the following geometric condition:

Assumption (A): There exists a direction $e_{2}$ parallel to $\partial L=\left\{x: x_{3}=0\right.$ or $\left.x_{3}=h\right\}$ and a point $O \in D$, such that if $O$ has the coordinate $x_{2}=0$, then

$$
x_{2} N_{2} \leq 0 \quad \text { on } \quad S,
$$

$N_{2}$ being the $x_{2}$-component of the normal $N$ which points into $D$.

Theorem 2.1. Let $k \notin \mathcal{K}$. If $u$ is a solution of the problem

$$
\begin{gathered}
\Delta u+k^{2} u=0 \quad \text { in } \quad D^{\prime}, \\
\left.u\right|_{\partial D^{\prime}=0,} \\
u \in L^{2}\left(D^{\prime}\right),
\end{gathered}
$$

then $u \equiv 0$ in $D^{\prime}$. The same conclusion holds if (2.3) is replaced by the condition

$$
\lim _{r \rightarrow \infty} \int_{\Gamma_{r}}\left[|u|^{2}+\left|u_{r}\right|^{2}\right] d s=0
$$


Proof. We start with the identity

$$
0=x_{2} u_{2}\left(u_{j j}+k^{2} u\right)=\left(x_{2} u_{2} u_{j}\right)_{j}+\left(\frac{k^{2} u^{2} x_{2}}{2}-\frac{x_{2}(\nabla u)^{2}}{2}\right)_{2}+\frac{(\nabla u)^{2}-k^{2} u^{2}}{2}-u_{2}^{2}
$$

where $u_{j}:=\partial u / \partial x_{j}$, and over the repeated indices summation is understood. Integrating (2.4) by parts over the region $D_{r}$ we get

$$
0=\int_{\partial D_{r}}\left(x_{2} u_{2} u_{j} N_{j}+\frac{k^{2} u^{2}-(\nabla u)^{2}}{2} x_{2} N_{2}\right) d s+\int_{D_{r}}\left(\frac{(\nabla u)^{2}-k^{2} u^{2}}{2}-u_{2}^{2}\right) d x .
$$

One has $N_{2}=0$ on $\partial L_{r}$, and since $u=0$ on $S \cup \partial L_{r}$, it follows that $\nabla u=N u_{N}$ and $u_{j}=N_{j} u_{N}$ on $S \cup \partial L_{r}$. According to Lemma 2.3 (see below) we have

$$
\int_{D^{\prime}}\left(|\nabla u|^{2}-k^{2}|u|^{2}\right) d x=0
$$

Therefore, taking $r \rightarrow \infty$ in (2.5), we get

$$
\int_{D^{\prime}} u_{2}^{2} d x=\int_{S} x_{2} N_{2}\left(u_{N}^{2}-\frac{u_{N}^{2}}{2}\right) d s+\lim _{r \rightarrow \infty} \int_{\Gamma_{r}} x_{2}\left(u_{2} u_{N}+\frac{k^{2} u^{2}-|\nabla u|^{2}}{2} N_{2}\right) d s .
$$

Furthermore, from Lemma 2.2 below it follows that

$$
|u|+|\nabla u| \leq c \exp (-\epsilon|\widehat{x}|), \quad \epsilon>0 .
$$

Therefore the second integral in (2.7) tends to zero as $r \rightarrow \infty$, and we get

$$
0 \leq \int_{D^{\prime}} u_{2}^{2} d x=\frac{1}{2} \int_{S} u_{N}^{2} x_{2} N_{2} d s
$$

which, because of the geometric assumption (A), implies $u_{N}=0$ on $S$. From this and (2.2) we have $u=u_{N}=0$ on $S$. By the uniqueness of the solution to the Cauchy problem for elliptic equations, one gets $u=0$ in $D^{\prime}$.

We prove now lemmas 2.2 and 2.3 .

Lemma 2.2. If $u$ solves (2.1)-(2.3), or (2.1)-(2.3'), then (2.8) holds.

Proof. Let us write

$$
u\left(\widehat{x}, x_{3}\right)=\sum_{m=1}^{\infty} \phi_{m}\left(x_{3}\right) u_{m}(\widehat{x}), \quad r:=|\hat{x}|>r_{0},
$$


where

$$
u_{m}(\widehat{x}):=\int_{0}^{h} u\left(\widehat{x}, x_{3}\right) \phi_{m}\left(x_{3}\right) d x_{3} .
$$

Then (2.1) and (2.10) imply

$$
\widehat{\Delta} u_{m}+k_{m}^{2} u_{m}=0 \text { in } \mathbb{R}^{2}, \quad k_{m}^{2}:=k^{2}-\lambda_{m}^{2},
$$

where $\widehat{\Delta}=\partial_{x_{1}}^{2}+\partial_{x_{2}}^{2}$.

By the assumption $k \in \mathcal{K}, k_{m} \neq 0$. Therefore, if (2.3) holds then

$$
\sum_{m=1}^{\infty} \int_{\Omega_{0}}\left|u_{m}\right|^{2} d \widehat{x}<\infty
$$

where $\Omega_{0}$ was defined above formula (1.1).

If (2.3') holds, then each $u_{m}$ satisfies condition (2.3'), because the functions $\phi_{m}$ are orthogonal.

Thus, (2.10), (2.12) and (2.13) or (2.13') imply that

$$
u_{m}=0 \quad \text { if } \quad k^{2}>\lambda_{m}^{2}
$$

and

$$
\left|u_{m}\right|+\left|\nabla u_{m}\right| \leq c \exp (-\epsilon|\widehat{x}|), \quad \epsilon>0, \quad \text { if } \quad k^{2}<\lambda_{m}^{2}
$$

The conclusions (2.14) and (2.15) follow from (2.10) since

$$
u_{m}(\widehat{x})=\sum_{l=-\infty}^{\infty}\left(c_{l}^{(1)} H_{|l|}^{(1)}\left(k_{m} r\right)+c_{l}^{(2)} H_{|l|}^{(2)}\left(k_{m} r\right)\right) \exp (i l \theta)
$$

if $k_{m}=\left(k^{2}-\lambda_{m}^{2}\right)^{1 / 2}>0$. Here $r=|\widehat{x}|>r_{0}, D \subset D_{r_{0}}, H_{l}^{(j)}(r), j=1,2$ are the Hankel functions and $c_{l}^{(j)}$ are arbitrary complex constants. From the asymptotics of Hankel functions as $r \rightarrow \infty$, it follows that (2.13) and (2.16) imply $c_{l}^{(j)}=0$ for all $l$ and $j=1,2$, and $m$ such that $k^{2}>\lambda_{m}^{2}$. Thus (2.14) holds.

If $k^{2}<\lambda_{m}^{2}$, that is $k_{m}=i\left|k_{m}\right|$, the Hankel function $H_{l}^{(1)}\left(i\left|k_{m}\right| r\right)$ decay exponentially as $r \rightarrow+\infty$ if $\left|k_{m}\right| \geq \epsilon>0$ and $H_{l}^{(2)}\left(i\left|k_{m}\right| r\right)$ grow exponentially as $r \rightarrow \infty$. Thus $c_{l}^{(2)}=0$ for all $l$ and (2.15) is established with $\epsilon=\min \left\{\left|k_{m}\right|: k^{2}<\lambda_{m}^{2}\right\}$. Lemma 2.2 is proved.

Let us now consider different boundary conditions on $\partial L$ and $S$. Assume that (2.2) is replaced by

$$
\begin{gathered}
u=0 \quad \text { at } \quad \partial L_{+}:=\left\{x \in L: x_{3}=h\right\} \\
\partial_{x_{3}} u=0 \text { at } \partial L_{-}:=\left\{x \in L: x_{3}=0\right\} \\
u=0 \text { on } S, \text { or } \quad u_{N}=0 \text { on } S .
\end{gathered}
$$


Then the arguments above should be changed only in the treatment of the integral over $\partial D_{r}$ in (2.5). Namely, at $L_{-}$one has $u_{N}=0$ and on $\partial L$ one has $N_{2}=0$ as before. The integral over $\Gamma_{r}$ vanishes, as $r \rightarrow \infty$, as before. Therefore only the integral over $S$ should be investigated for the boundary conditions (2.19). The Dirichlet boundary condition (2.19) has been investigated already in the proof of Theorem 2.1. Consider the Neumann boundary condition. Equation (2.5) now yields:

$$
\int_{D^{\prime}} u_{2}^{2} d x=\frac{1}{2} \int_{S} x_{2} N_{2}\left[k^{2} u^{2}-(\nabla u)^{2}\right] d s=: I .
$$

If $u_{N}=0$ on $S$, then $\nabla u=\nabla_{t} u$, where $\nabla_{t} u$ is the gradient in the tangential to $S$ plane orthogonal to $N$. Thus, the integral $I$ in (2.20) can be written as

$$
I=\frac{1}{2} \int_{S} x_{2} N_{2}\left[k^{2} u^{2}-\left(\nabla_{t} u\right)^{2}\right] d s .
$$

If Assumption (A) holds, then one could conclude that $I \leq 0$ and, therefore, could obtain a uniqueness theorem from equation (2.20), provided that

$$
k^{2} u^{2} \geq\left(\nabla_{t} u\right)^{2} \quad \text { on } S,
$$

for the solution to (2.1) satisfying the second boundary condition (2.19).

However, this is not possible since inequality (2.22) does not hold in general.

So, it is an open problem to find out when a uniqueness theorem, analogous to Theorem 2.1, holds in the case of acoustically hard obstacle, that is for the Neumann boundary condition on $S$. In fact, examples of non-uniqueness are discussed in Refs. $2,2 \mathrm{a}$ and 3 .

Let us now prove Lemma 2.3.

Lemma 2.3. If $u$ satisfies conditions (2.1)-(2.3) then $\nabla u \in L^{2}\left(D^{\prime}\right)$ and (2.15) holds.

Proof. From (2.1) and Green's formula we get

$$
0=\int_{D_{r}} \bar{u}\left(\Delta+k^{2}\right) u d x=\int_{D_{r}}\left(k^{2}|u|^{2}-|\nabla u|^{2}\right) d x+\int_{\partial D_{r}} \bar{u} u_{N} d s .
$$

Thus

$$
\int_{D_{r}}\left(|\nabla u|^{2}-k^{2}|u|^{2}\right) d x=\int_{\partial D_{r}} \bar{u} u_{N} d s:=I .
$$

If boundary conditions (2.2) (or (2.17)-(2.19)) hold, then

$$
I=I(r):=\int_{\Gamma_{r}} \bar{u} u_{N} d s=\int_{\Gamma_{r}} \bar{u} u_{r} d s,
$$


where $\Gamma_{r}:=\{x:|\hat{x}|=r\}$.

Lemma 2.2 is proved if we prove that

$$
I\left(r_{n}\right) \rightarrow 0 \quad \text { as } \quad r_{n} \rightarrow \infty
$$

for some sequence $r_{n}$.

If (2.3') holds, then (2.26) is obvious. Suppose (2.3) holds and we want to prove (2.26). Let

$$
w(r):=\int_{\Gamma_{r}}|u|^{2} r d \theta d x_{3}
$$

Then

$$
w^{\prime}(r):=\frac{d w}{d r}=2 \operatorname{Re} I(r)+\frac{1}{r} w(r),
$$

so

$$
2 \operatorname{Re} I(r)=w^{\prime}(r)-\frac{1}{r} w(r)
$$

Since $u$ solves (2.1), (2.2), Green's formula yields

$$
0=\int_{\Gamma_{r}}\left(\bar{u} u_{r}-\overline{u_{r}} u\right) d s=2 \operatorname{Im} \int_{\Gamma_{r}} \bar{u} u_{r} d s .
$$

Thus $\operatorname{Im} I(r)=0$ and $(2.29)$ can be written as

$$
I(r)=\frac{1}{2}\left(w^{\prime}(r)-\frac{1}{r} w(r)\right)
$$

Lemma 2.3 follows from (2.31), and the following Lemma 2.4.

Lemma 2.4. Assume $w(r) \geq 0, \int_{a}^{\infty} w(r) d r<\infty, w \in C^{2}(a, \infty), a=$ const $\geq 0$. Then there is a sequence $r_{n} \rightarrow \infty$ such that

$$
\left|w^{\prime}\left(r_{n}\right)\right|+\left|w\left(r_{n}\right)\right| \rightarrow 0
$$

Proof. If $\int_{a}^{\infty} w(r) d r<\infty$ and $w(r) \geq 0$ decreases monotonically to zero in a neighborhood of infinity, then $w^{\prime}(r)<0$ and $w^{\prime}\left(r_{n}\right) \rightarrow 0$ for some sequence $r_{n} \rightarrow \infty$, since $\sup _{r>a} \int_{a}^{r} w^{\prime}(r) d r<\infty$.

If $w(r)$ does not decrease monotonically to zero near infinity, then $w(r)$ has infinitely many nonnegative local maxima and minima. Let $w\left(r_{n}\right)$ are local minima of $w(r), r_{n} \rightarrow \infty$. Then $w^{\prime}\left(r_{n}\right)=0$ and one can select a subsequence $r_{n}^{\prime}$ out of the sequence $r_{n}$, such that $w\left(r_{n}^{\prime}\right) \rightarrow 0$ as $r_{n}^{\prime} \rightarrow \infty$. Indeed, since $\int_{a}^{\infty} w(r) d r<\infty$, if such a subsequence would not exist, then, for some $\epsilon>0$, one would have $w\left(r_{n}\right) \geq \epsilon>0$ as $r_{n} \rightarrow \infty$. Then $w(r) \geq \epsilon$ for $r \geq r_{1}$ and this contradicts the assumptions $\int_{a}^{\infty} w(r) d r<\infty, w(r) \geq 0$. Lemma 2.4 is proved.

Remark 2.1: Consider a compact perturbation of the boundary $\partial L$ which is denoted $\Sigma$. Assume that $S \subset L$ and that the region $L_{\Sigma}$, which is the layer with 
the compactly perturbed boundary, satisfies the Assumption (A). Then Theorem 1 holds in $L_{\Sigma}$ with boundary condition $\left.u\right|_{\Sigma}=0$. The proof is the same as above.

Remark 2.2: Similar results hold for an obstacle in a cylindrical waveguide and for a compact perturbation of the boundary of the cylinder provided that the perturbed cylinder lies inside the unperturbed one and Assumption (A) holds for the perturbed cylinder.

Recently AGR found another geometrical assumption and a short proof of the uniqueness theorem similar to Theorem 2.1.

Assumption (B):

There exists a point $O$ inside $D$ such that if $O$ is the origin, and $N$ is the unit normal to $S$ pointing into $D$, then

$$
x \cdot N \leq 0 \quad \forall x \in S .
$$

This assumption holds, in particular, for convex obstacles.

Theorem 2.1'. The conclusion of Theorem 2.1 holds under the assumption (B).

Proof. The proof is based on the well-known Rellich's identity:

$$
\int_{S}\left(2 x \cdot \nabla u u_{N}-x \cdot N|\nabla u|^{2}\right) d s=2 \int_{D} x \cdot \nabla u \Delta u d x+(2-n) \int_{D}|\nabla u|^{2} d x .
$$

Here $n$ is the dimension of the space and one may assume without loss of generality that $u$ is real-valued in the proof of Theorems 2.1 and 2.1'.

If $u=0$ on $S$ then $\nabla u=N u_{N}$ on $S$. Equation (2.1) and the Rellich's identity yield:

$\int_{S} x \cdot N u_{N}^{2} d s=-k^{2} \int_{D^{\prime}}\left[\left(x_{j} u^{2}\right)_{j}-n u^{2}\right] d x+(2-n) \int_{D^{\prime}}|\nabla u|^{2} d x=2 \int_{D^{\prime}}|\nabla u|^{2} d x>0$.

Here we have used (2.6) and (2.2). Assumption (B) and the last equation imply $u=0$ in $D^{\prime}$. Theorem $2.1^{\prime}$ is proved.

\section{Boundary value problems in $D^{\prime}$}

In this section we investigate the boundary value problem

$$
\begin{gathered}
\left(\Delta+k^{2}\right) u=f \quad \text { in } \quad D^{\prime}, \quad f \in L_{0}^{2}\left(D^{\prime}\right), \quad k \notin \mathcal{K} \\
\left.u\right|_{S}=\phi(s),\left.\quad u\right|_{\partial L}=0 \quad \text { or }\left.\quad u\right|_{L^{+}}=\left.0 \quad u_{N}\right|_{L^{-}}=0, \\
u=\sum_{m=1}^{\infty} \phi_{m}\left(x_{3}\right) u_{m}(\widehat{x}), \quad \widehat{x}=\left(x_{1}, x_{2}\right), \quad r>r_{0},
\end{gathered}
$$

where $r_{0}$ was defined above formula (1.1), and $u_{m}$ satisfy the radiation conditions 


$$
\begin{gathered}
\sqrt{r}\left(u_{m r}-i k_{m} u_{m}\right) \underset{r \rightarrow \infty}{\longrightarrow} 0, \quad r=|\widehat{x}|, \quad k_{m}=\left(k^{2}-\lambda_{m}^{2}\right)^{1 / 2}>0 \\
\left|u_{m}\right|=O(\exp (-\epsilon r)) \quad \text { if } \quad k^{2}<\lambda_{m}^{2}, \quad \epsilon>0 .
\end{gathered}
$$

Theorem 3.1. For any $f \in L_{0}^{2}\left(D^{\prime}\right)$ and any $\phi \in H^{3 / 2}(S)$, the problem (3.1)-(3.5) has a unique solution.

Proof. Uniqueness follows from Theorem 2.1. Indeed, if $u$ solves homogeneous equations (3.1) and (3.2), then

$$
\lim _{r \rightarrow \infty} \int_{\Gamma_{r}}\left(\bar{u} u_{r}-\overline{u_{r}} u\right) d s=0,
$$

and because the system of functions $\phi_{m}$ is orthogonal, it follows that each $u_{m}$ satisfies the above relation as well. This, and the radiation condition (3.4) imply that each of $u_{m}$ satisfy condition (2.3').

The equation

$$
\Delta u+k_{m}^{2} u_{m}=0 \quad \text { in } \quad \Omega_{0}, k_{m}>0
$$

and condition (2.3') for $u_{m}$ imply that $u_{m}=0$ in $\Omega_{0}$, (see Ref. 10, p.25). If $\lambda_{m}^{2}>k^{2}$, then condition (3.5) says that $u_{m}$ decay exponentially. Therefore any solution $u$ to the homogeneous problem (3.1)-(3.5) belongs to $L^{2}\left(D^{\prime}\right)$ and solves (2.1)-(2.3). By Theorem 2.1, this $u=0$ in $D^{\prime}$, as claimed.

Existence is a consequence of the uniqueness and of the Fredholm property of the problem (3.1)-(3.5). Let us establish this property.

First, we introduce the function

$$
w:=u-\int_{D^{\prime}} g(x, y, k) f(y) d y
$$

where $g$ is the Green function of the Helmholtz operator in $L$. If, for example, (1.5) is assumed, then

$$
g(x, y, k)=\sum_{m=1}^{\infty} \frac{2}{h} \sin \left(m \pi x_{3} / h\right) \sin \left(m \pi y_{3} / h\right) \frac{i}{4} H_{0}^{1}\left(k_{m}|\hat{x}-\hat{y}|\right) .
$$

The function $w$ solves the problem

$$
\begin{gathered}
\left(\Delta+k^{2}\right) w=0 \quad \text { in } \quad D^{\prime} \\
\left.w\right|_{\partial L}=0,\left.\quad w\right|_{S}=h:=\phi-\int_{D^{\prime}} g(s, y, k) f(y) d y
\end{gathered}
$$

and it satisfies (3.3)-(3.5). 
Therefore, one may assume $f=0$ in (3.1) without loss of generality but then one has to consider the nonhomogeneous boundary condition (3.8). We look for the solution to (3.7)-(3.8) in the form

$$
w(x)=\int_{S} g(x, t, k) \mu(t) d t, \quad x \in D^{\prime} .
$$

The function (3.9) satisfies (3.7) and the first condition (3.8) for any $\mu(t)$. It will also satisfy the second condition (3.8), if

$$
Q(k) \mu:=\int_{S} g(x, t, k) \mu(t) d t=h(x), \quad x \in S .
$$

The operator $Q(k)$ in $(3.10)$ acts from $H^{l}(S) \rightarrow H^{l+1}(S)$ for any positive integer $l$ (Ref. 10, p.81) provided that $S$ is sufficiently smooth. For our purposes, $l=0$ is sufficient, and then Lipschitz $S$ is admissible. However, in the argument below we use the space $H^{2}(S)$, and because of this we assume that $S$ is $C^{2}$. For a theory with Lipschitz $S$ see Refs 15 and 17.

By changing $g$ to $g_{\rho}$ if necessary, one can always assume that $Q$ or $Q_{\rho}$ is injective. Here $Q_{\rho} \mu:=\int_{S} Q_{\rho} \mu d t$ and $g_{\rho}$ is the Green function in $L \backslash B_{\rho}, B_{\rho}$ being a ball of small radius $\rho, B_{\rho} \subseteq D$ (see Ref. 10, pp. 20, 29). If $Q$ is invertible, then the map $Q: H^{l}(S) \rightarrow H^{l+1}(S)$ is equivalent to a Fredholm map on $H^{l}(S)$. Indeed, take $k_{1} \neq k$ and put $Q_{1}:=Q(k)$. Let $Q_{1}$ be injective. Then $Q=Q-Q_{1}+$ $Q_{1}=Q_{1}\left[I+Q_{1}^{-1}\left(Q-Q_{1}\right)\right]$. The operator $Q_{1}^{-1}\left(Q-Q_{1}\right)$ is compact in $H^{l}(S)$ since $Q-Q_{1}: H^{l} \rightarrow H^{l+2}$ while $Q_{1}^{-1}: H^{l+1} \rightarrow H^{l}$. Thus $Q_{1}^{-1} Q$ is a Fredholm operator equivalent to $Q$ (see, e.g. Refs. 10 and 14). Theorem 2.1 yields injectivity of $Q_{\rho}$ for a suitable choice of $\rho$. Thus, the corresponding equation (3.10) is uniquely solvable for $Q$ replaced by $Q_{\rho}$. If $Q$ is not injective, the above argument shows that it has a finite dimensional null-space and equation (3.10) is solvable, if $h$ satisfies finitely many orthogonality relations.

However, the boundary value problem (3.1)-(3.5) is uniquely and unconditionally solvable for any $f$ and $\psi$, the solution given by

$$
\begin{aligned}
& u(x)=\int_{D^{\prime}} g(x, y, k) f(y) d y+w(x), \\
& w(x)=\int_{S} g_{\rho}(x, t, k)\left(Q_{\rho}{ }^{-1} h\right)(t) d t,
\end{aligned}
$$

for a properly chosen small $\rho>0$, as our argument above shows.

Other methods can be also used for proving existence of the solution to the boundary value problem (3.1)-(3.5) (see, e.g. Refs. 10,15). One can also treat the case when $k^{2}$ is replaced by $k^{2} n\left(x_{3}\right)$ (horizontally stratified waveguide). In this case the eigenvalues (1.7) and (1.8) and the eigenfunctions (1.9) and (1.10) will be different, but the scheme of the study remains essentially the same. 


\section{Resolvent kernel of the Laplacian and limiting absorption principle}

Let us now consider the boundary value problem

$$
\begin{gathered}
\left(\Delta+k^{2}+i \epsilon\right) G_{\epsilon}(x, y)=-\delta(x-y) \quad \text { in } \quad D^{\prime}, \quad k \notin \mathcal{K} \\
\left.G_{\epsilon}\right|_{S}=0,\left.\quad G_{\epsilon}\right|_{\partial L}=0, \quad \text { or }\left.\quad G_{\epsilon}\right|_{\partial L_{+}}=0,\left.\quad G_{\epsilon N}\right|_{\partial L_{-}}=0, \\
G_{\epsilon} \in L^{2}\left(D^{\prime}\right) .
\end{gathered}
$$

This problem is uniquely solvable if $\epsilon>0$ (Ref. 10, p. 309).

Theorem 4.1. The limit of $G_{\epsilon}(x, y)$, as $\epsilon \rightarrow 0$, exists in the sense of uniform convergence on compact subsets $\left\{(x, y, k): 0<R_{1} \leq|x-y| \leq R_{2}, a<k<\right.$ $b,(a, b) \cap \mathcal{K}=\emptyset\}$, where $R_{1}, R_{2}$ are some positive constants.

Proof. To prove this limiting absorption principle we start with the equations

$$
G_{\epsilon}(x, y)=g_{\epsilon}(x, y)-\int_{S} g_{\epsilon}(x, t, k) \mu_{\epsilon} d t, \quad \mu_{\epsilon}=\frac{\partial}{\partial N_{t}} G_{\epsilon}(t, y),
$$

where $g_{\epsilon}(x, y)$ is the Green function for the Helmholtz operator in $L$ and $N_{t}$ is the normal to $S$ pointing into $D^{\prime}$.

Differentiating the integral in (4.4) along the normal to $S$, and using the standard formulas for the exterior normal derivative of the single layer potentials, we get

$$
\mu_{\epsilon}+A_{\epsilon} \mu_{\epsilon}=2 \frac{\partial g_{\epsilon}}{\partial N_{s}}, \quad A_{\epsilon} \mu=2 \int_{S} \frac{\partial g_{\epsilon}(s, t)}{\partial N_{s}} \mu(t) d t
$$

The operator $A_{\epsilon}$ is compact in $L^{2}(S)$ for any $0 \leq \epsilon \leq \epsilon_{0}, \epsilon_{0}$ being a positive number. Consequently, the operator $I+A_{\epsilon}$ is Fredholm and it depends continuously (in the operator norm) on $\epsilon, 0 \leq \epsilon \leq \epsilon_{0}$. For each $\epsilon \in\left[0, \epsilon_{0}\right]$ the operator $I+A_{\epsilon}$ is injective, as we will prove later. Therefore

$$
\sup _{0 \leq \epsilon \leq \epsilon_{0}}\left\|\left(I+A_{\epsilon}\right)^{-1}\right\| \leq c<\infty,
$$

where $c>0$ is a constant, and

$$
\mu_{\epsilon} \underset{L^{2}(S)}{\longrightarrow} \mu_{0}:=\left(I+A_{0}\right)^{-1} 2 \frac{\partial g(s, t, k)}{\partial N_{s}},
$$

where $g(x, s, k)$ is the limit of $g_{\epsilon}$ at $\epsilon=0$.

Thus, as $\epsilon \rightarrow 0$, one gets

$$
G_{\epsilon} \rightarrow G:=g(x, y, k)-\int_{S} g(x, s, k) \mu_{0}(s, y, k) d s .
$$


To complete the proof we need to prove (4.6). The continuity of the inverse operator $\left(I+A_{\epsilon}\right)^{-1}$ with respect to $\epsilon$ follows from (4.6) and the identity

$$
A^{-1}-B^{-1}=B^{-1}(B-A) A^{-1}
$$

There are two claims to prove:

(a) $\left(I+A_{0}\right)^{-1}$ exists, and

(b) the estimate (4.6) holds.

Let us assume (a) and prove (4.6). Suppose the contrary, that is

$$
\sup _{0 \leq \epsilon \leq \epsilon_{0}}\left\|\left(I+A_{\epsilon}\right)^{-1}\right\|=\infty
$$

Then there is a sequence $\epsilon_{n} \rightarrow \widetilde{\epsilon}, 0 \leq \widetilde{\epsilon} \leq \epsilon_{0}$, such that $\left\|\left(I+A_{\epsilon_{n}}\right)^{-1}\right\| \rightarrow \infty$. Since

$$
F_{n}:=\left(I+A_{\epsilon_{n}}\right)^{-1}=\left(I+A_{\widetilde{\epsilon}}\right)^{-1}+\left(I+A_{\widetilde{\epsilon}}\right)^{-1}\left(A_{\widetilde{\epsilon}}-A_{\epsilon_{n}}\right)\left(I+A_{\epsilon_{n}}\right)^{-1},
$$

and

$$
\left\|A_{\tilde{\epsilon}}-A_{\epsilon_{n}}\right\| \rightarrow 0, \quad \text { as } \quad n \rightarrow \infty
$$

one gets

$$
\left(I+T_{n}\right) F_{n}=\left(I+A_{\tilde{\epsilon}}\right)^{-1}
$$

where

$$
T_{n}:=-\left(I+A_{\tilde{\epsilon}}\right)^{-1}\left(A_{\tilde{\epsilon}}-A_{\epsilon_{n}}\right), \quad\left\|T_{n}\right\| \rightarrow 0 \quad \text { as } \quad n \rightarrow \infty .
$$

Thus

$$
\left\|F_{n}\right\| \leq\left\|\left(I+T_{n}\right)^{-1}\right\|\left\|\left(I+A_{\epsilon}^{\sim}\right)^{-1}\right\| \leq c
$$

where $c$ does not depend on $n$. This contradiction proves (4.6).

It remains to be proved that $\left(I+A_{\epsilon}\right)^{-1}$ exists for all $\epsilon \in\left[0, \epsilon_{0}\right]$. Since the operators $I+A_{\epsilon}$ are Fredholm-type, it is sufficient to check that its null-space is trivial. Assume that

$$
\left(I+A_{\epsilon}\right) \mu=0, \quad \mu \not \equiv 0 .
$$

Then the function $w_{\epsilon}:=\int_{S} g_{\epsilon}(x, t) \mu(t) d t$ solves the problem

$$
\left(\nabla^{2}+k^{2}+i \epsilon\right) w_{\epsilon}=0 \quad \text { in } \quad D \cup D^{\prime}, \quad w_{\epsilon N}^{+}=0 \quad \text { on } S,
$$

where $w_{\epsilon N}^{+}$is the limiting value on $S$ of the normal derivative from inside of $D$. Thus $w_{\epsilon}=0$ in $D$, if $\epsilon>0$ and $w_{\epsilon}=0$ on $S$. On the other hand, the solution $w_{\epsilon}$ of the problem

$$
\left(\nabla^{2}+k^{2}+i \epsilon\right) w_{\epsilon}=0 \quad \text { in } \quad D^{\prime}, \quad w_{\epsilon}=0 \quad \text { on } \quad S, \quad w_{\epsilon} \in L^{2}\left(D^{\prime}\right)
$$

vanishes in $D^{\prime}$. Thus, if $\epsilon>0, w_{\epsilon}=0$ in $D \cup D^{\prime}$, and $\mu=w_{\epsilon N}^{+}-w_{\epsilon N}^{-}=0$, where $w_{\epsilon N}^{-}$is the limiting value of the normal derivative on $S$ from outside of $D$. 
If $\epsilon=0$, the above argument is valid for $k^{2} \notin \sigma\left(\Delta_{N}(D)\right)$, that is, $k^{2}$ is not a Neumann eigenvalue of the Laplacian in $D$, and yields the conclusion $w_{0}=0$ in $D$. To get the conclusion $w_{0}=0$ in $D^{\prime}$, one notes that $w_{0}$ solves the homogeneous problem (3.1)-(3.5) and therefore Theorem 3.1 implies the desired conclusion.

In case $k^{2} \in \sigma\left(\Delta_{N}(D)\right)$, one should repeat the above argument with $g$ replaced by $g_{\rho}$ (defined in section 3, in the second paragraph below equation (3.10)), where $\rho>0$ is chosen so that $k^{2} \notin \sigma\left(\Delta_{N}\left(D \backslash B_{\rho}\right)\right)$. Such a choice is always possible (see Ref. 10). Therefore, the limiting absorption principle for $G(x, y, k)$ is established.

A proof of this principle, based on the compactness argument, can also be given ( see Refs. 9 and 10).

\section{Scattering solutions}

We define the scattering solutions using the approach introduced in Ref. 16 (see also Ref. 10, pp. 46-48). If $G(x, y, k)$ is the resolvent kernel of the Laplacian in $D^{\prime}$, then we study the limit of $G$ as $|\widehat{y}|:=r \rightarrow \infty, \widehat{y} / r=-\alpha, \alpha \in S^{1}, S^{1}$ being the unit circle, $\widehat{y}:=\left(y_{1}, y_{2}\right)$. It follows from (4.8) and the symmetry of the Green function $G(x, y, k)=G(y, x, k)$, that, as $r \rightarrow \infty$ and $\widehat{y} / r=-\alpha$,

$$
G(x, y, k) \sim \sum_{m=1}^{\infty} \gamma_{m}(r) \phi_{m}\left(y_{3}\right) \psi_{m}\left(x, \alpha, k_{m}\right)
$$

where

$$
\gamma_{m}(r):=\frac{i}{4} \sqrt{\frac{2}{\pi k_{m} r}} \exp \left(i k_{m} r-\frac{i \pi}{4}\right),
$$

and $k_{m}=\left(k^{2}-\lambda_{m}^{2}\right)^{1 / 2} \quad$ if $\quad k^{2}>\lambda_{m}^{2}, \quad k_{m}=i\left(\lambda_{m}^{2}-k^{2}\right)^{1 / 2} \quad$ if $\quad k^{2}<\lambda_{m}^{2}$, the square roots are chosen so that $\sqrt{t}>0$ if $t>0$.

The scattering solutions are the coefficients $\psi_{m}$ in (5.1), defined by

$$
\psi_{m}:=\phi_{m}\left(x_{3}\right) \exp \left(i k_{m} \alpha \cdot \widehat{x}\right)-\int_{S} \phi_{m}\left(t_{3}\right) \exp \left(i k_{m} \alpha \cdot t\right) \mu_{0}(t, x, k) d t:=\psi_{m}^{(0)}+v_{m},
$$

where $\mu_{0}(t, x, k)$ is defined in equation (4.8), and

$\psi_{m}^{(0)}\left(x, \alpha, k_{m}\right)=\phi_{m}\left(x_{3}\right) u_{m}^{(0)}\left(\widehat{x}, \alpha, k_{m}\right), u_{m}^{(0)}\left(\widehat{x}, \alpha, k_{m}\right)=\exp \left(i k_{m} \alpha \cdot \widehat{x}\right) \quad$ if $\quad k^{2}>\lambda_{m}^{2}$.

If the obstacle is absent, then, as $r \rightarrow \infty, \widehat{y} / r=-\alpha$,

$$
g(x, y, k) \sim \sum_{m=1}^{\infty} \gamma_{m}(r) \phi_{m}\left(y_{3}\right) \psi_{m}^{(0)}\left(\widehat{x}, \alpha, x_{3}, k_{m}\right)
$$

where $g$ is the resolvent kernel of the Laplacian in $L$ in the absence of $D$.

The asymptotics (5.1) and (5.5) are obtained by taking asymptotics termwise in the series for $G$ and $g$ respectively. 
The series (5.5) converges for $x_{3} \neq y_{3}$, and also for $x_{3}=y_{3}$ provided that $|\alpha \cdot x|<|x|$. We do not claim that these series converge pointwise for all $\widehat{x} \in \mathbb{R}^{2}$. Indeed, if $k^{2}<\lambda_{m}^{2}$, then the corresponding term in the expansion of $g(x, y, k)$ is

$$
\phi_{m}\left(x_{3}\right) \phi_{m}\left(y_{3}\right) \frac{i}{4} H_{0}^{(1)}\left(k_{m}|\widehat{x}-\widehat{y}|\right), \quad k_{m}=i\left(\lambda_{m}^{2}-k^{2}\right)^{1 / 2},
$$

and its asymptotics as $r=|\widehat{y}| \rightarrow \infty, \widehat{y} / r=-\alpha$, is

$$
\phi_{m}\left(x_{3}\right) \phi_{m}\left(y_{3}\right) \frac{i}{4} e^{-\frac{i \pi}{4}} \sqrt{\frac{2}{\pi k_{m} r}} \exp \left(-\left|k_{m}\right| r\right) \exp \left(-\left|k_{m}\right| \alpha \cdot \widehat{x}\right),
$$

where $\alpha \cdot \widehat{x}=\alpha_{1} x_{1}+\alpha_{2} x_{2}$. The last expression increases exponentially as $|\widehat{x}| \rightarrow \infty$, if $\alpha \cdot \widehat{x}<0$. However, if $r>|\widehat{x}|$, then

$$
-\left|k_{m}\right|(r+\alpha \cdot \widehat{x}) \leq-\left|k_{m}\right| \epsilon, \quad \epsilon>0 .
$$

Therefore the asymptotic series (5.5) converges uniformly on compact subsets of $\mathbb{R}^{2} \times S^{1} \times\left(\mathbb{R}_{+} \backslash \mathcal{K}\right)$. Note also that the functions $\psi_{m}(x)$ and $\psi_{m}^{(0)}(x)$ are not uniformly bounded in $D^{\prime}$. For example, the function $\psi_{m}^{(0)}(x)$ is exponentially growing as $|\widehat{x}| \rightarrow \infty, \alpha \cdot \widehat{x}>0, \lambda_{m}^{2}>k^{2}$. If $\lambda_{m}^{2}<k^{2}$, then $\psi_{m}^{(0)}(x)=\phi_{m}\left(x_{3}\right) \exp \left(i k_{m} \alpha \cdot \widehat{x}\right)$ is uniformly bounded in $D^{\prime}$.

Denote by $M$ the integer such that $\lambda_{m}^{2}<k^{2}$ if $m \leq M, \lambda_{m}^{2}>k^{2}$ if $m>M$. In the sequel, we consider as an incident field in $D^{\prime}$ the functions $\psi_{m}^{(0)}\left(x, \alpha, k_{m}\right)$, $m \leq M$. Fixing $m \leq M$, we formulate the scattering problem in $D^{\prime}$ as follows:

Find the solution $\psi_{m}$ to the problem

$$
\begin{gathered}
\left(\Delta+k^{2}\right) \psi_{m}=0 \quad \text { in } \quad D^{\prime}, \\
\left.\psi_{m}\right|_{L}=0 \quad\left(\text { or }\left.\psi_{m N}\right|_{L_{-}}=0,\left.\quad \psi_{m}\right|_{L_{+}}=0\right), \\
\psi_{m}=0 \quad \text { on } S, \\
\psi_{m}(x, k)=\psi_{m}^{(0)}\left(x, \alpha, k_{m}\right)+v_{m}(x, k),
\end{gathered}
$$

where $v_{m}(x, k)$ satisfies equation (5.6), the boundary conditions (5.7) on $L, v_{m}=$ $-\psi_{m}^{(0)}$ on $S$, and it admits the representation (analogous to (3.3))

$$
v_{m}(x, k)=\sum_{l=1}^{\infty} v_{m}^{l}(\hat{x}, k) \phi_{l}\left(x_{3}\right), \quad r>r_{0} .
$$

Here

$$
v_{m}^{l}(\widehat{x}, k)=\int_{0}^{h} v_{m}(x, k) \phi_{l}\left(x_{3}\right) d x_{3},
$$

and $v_{m}^{l}$ satisfy the radiation conditions

$$
\int_{S_{r}}\left|\frac{\partial v_{m}^{l}}{\partial r}-i k_{l} v_{m}^{l}\right|^{2} d s \underset{r \rightarrow \infty}{\longrightarrow} 0, \quad \text { if } \quad l \leq M
$$


and

$$
\left|v_{m}^{l}\right| \underset{r \rightarrow \infty}{\longrightarrow} 0, \quad \text { if } \quad l>M .
$$

Note that $v_{m}$ solves a problem analogous to (3.7)-(3.8). Thus, using a formula similar to (3.9), and formulas (5.1)-(5.5), we get the representation

$$
v_{m}(x, k)=-\int_{S} \phi_{m}\left(t_{3}\right) u_{m}^{(0)}\left(t, \alpha, k_{m}\right) \mu(t, x, k) d t,
$$

where $\mu:=\frac{\partial G(t, x, k)}{\partial N_{t}}, N_{t}$ being the normal to $S$ at the point $t$, for the case of Dirichlet boundary conditions on $\partial D^{\prime}$. Then $v_{m}^{l}$ are given by

$$
v_{m}^{l}(\widehat{x}, k)=-\int_{S} \phi_{m}\left(t_{3}\right) u_{m}^{(0)}\left(t, \alpha, k_{m}\right) \int_{0}^{h} \mu(t, x, k) \phi_{l}\left(x_{3}\right) d x_{3} d t .
$$

Note that, the representation (5.14) can be used in the numerical calculation of $v_{m}$, after solving the integral equation

$$
\mu+A \mu=2 \frac{\partial g}{\partial N}, \quad A \mu:=2 \int_{S} \frac{\partial g(s, t, k)}{\partial N_{s}} \mu(t) d t,
$$

$N_{s}$ being the normal to $S$ at $s$ pointing into $D^{\prime}$. Equation (5.16) is similar to (4.5).

Let us now state and prove the eigenfunction expansion theorem and sketch its proof following Ref. 17 and Ref. 10, p.48.

Theorem 5.1. $\quad$ Let $f \in L^{2}\left(D^{\prime}\right)$. Denote

$$
\hat{f}_{m}\left(\alpha, k_{m}\right):=\int_{D^{\prime}} f(x) \overline{\psi_{m}\left(x, \alpha, k_{m}\right)} d x, \quad m=1,2, \ldots,
$$

where $\psi_{m}\left(x, \alpha, k_{m}\right)$ are the scattering solutions (see (5.1), (5.3), and (5.6)-(5.13)).

Then

$$
f(x)=\frac{1}{4 \pi^{2}} \sum_{m=1}^{\infty} \int_{S^{1}} d \alpha \int_{0}^{\infty} d \xi \xi \hat{f}_{m}(\alpha, \xi) \psi_{m}(x, \alpha, \xi),
$$

Proof. The Dirichlet Laplacian has no spectrum in the interval $\left(-\infty, \lambda_{1}\right)$ (see Eq. (1.7)). Thus (see Ref. 10, p.48, Eq.(16)) we have

$$
f(x)=\frac{1}{i \pi} \int_{\lambda_{1}}^{\infty} d k k \int_{D^{\prime}} d y f(y)[G(x, y, k)-\overline{G(x, y, k)}],
$$

where the bar stands for complex conjugate.

Using Green's formula and formula (5.1), we express the $G-\bar{G}$ in terms of the scattering solutions:

$$
\begin{array}{r}
G(x, y, k)-\overline{G(x, y, k)}=\lim _{r \rightarrow \infty} \int_{|\hat{t}|=r} \int_{0}^{h} d t_{3} d \hat{t}\left[\overline{G(t, y, k)} G_{r}(x, t, k)\right. \\
\left.-G(x, t, k) \overline{G_{r}(t, y, k)}\right]:=J .
\end{array}
$$


From $(5.20),(5.1)$ and $(5.2)$, with $\alpha=-\frac{\hat{t}}{|\hat{t}|}$, we have

$$
\begin{aligned}
J=\sum_{m, l=1}^{\infty} \gamma_{m}(r) \overline{\gamma_{l}(r)} r\left(2 i k_{m}\right) \int_{0}^{h} d t_{3} \phi_{m}\left(t_{3}\right) \phi_{l}\left(t_{3}\right) \\
\times \int_{S^{1}} d \alpha \psi_{m}\left(x, \alpha, k_{m}\right) \overline{\psi_{l}\left(y, \alpha, k_{l}\right)}
\end{aligned}
$$

or

$$
J=\sum_{m=1}^{\infty} \frac{i}{4 \pi} \int_{S^{1}} \psi_{m}\left(x, \alpha, k_{m}\right) \overline{\psi_{m}\left(y, \alpha, k_{m}\right)} d \alpha .
$$

Here we have used the orthogonality of $\phi_{m}$ and the conditions $\Im \gamma_{m}=0, \quad \Im \psi_{m}=0$ if $k^{2}<\lambda_{m}^{2}$. These equations imply that, for each $m=1,2,3, \ldots$, the interval $k^{2}<\lambda_{m}^{2}$ does not contribute to the eigenfunction expansion. Let $\xi^{2}:=k^{2}-\lambda_{m}^{2}, k d k=\xi d \xi$. From (5.20), (5.21) and (5.22), we get the eigenfunction expansion formula (5.18) where $\hat{f}_{m}\left(\alpha, k_{m}\right)$ is defined in (5.17). Theorem 5.1 is proved.

The result in Theorem 5.1 is similar to the main result by Goldstein ${ }^{4}$, for cylindrical waveguides, but our proof is quite different, much shorter, and, as mentioned above, it follows the proof in Refs. 16 and 10.

\section{Inverse scattering problems}

Suppose that two obstacles, $D_{1}$ and $D_{2}$, generate the same scattering data. We want to derive from this that $D_{1}=D_{2}$. We assume that the boundary condition on $D_{1}$ and $D_{2}$ is the Dirichlet one. As the scattering data we take the partial scattering amplitudes $A_{m l}\left(\alpha, \alpha^{\prime}, k_{m}, k_{l}\right), l \leq M$, for all $\alpha, \alpha^{\prime} \in S^{1}$. Here $m, 1 \leq m \leq M$, is assumed fixed. In other words, we send an incident wave $\psi_{m}^{(0)}(x, \alpha)$ and we observe the (partial) scattering amplitudes $A_{m l}\left(\alpha, \alpha^{\prime}, k_{m}, k_{l}\right), 1 \leq l \leq M$, defined as the coefficients in the asymptotics

$$
v_{m}^{l}(\widehat{x}, k) \sim A_{m l}\left(\alpha, \alpha^{\prime}, k_{m}, k_{l}\right) \gamma_{l}(|\widehat{x}|), \quad|\widehat{x}| \rightarrow \infty, \quad \frac{\widehat{x}}{|\widehat{x}|}=\alpha^{\prime},
$$

where $v_{m}^{l}(\widehat{x}, k)$ are defined in (5.11). Using the now standard arguments (Ref. 10, pp. 84-88) and the uniqueness Theorem 1.1, we prove the following uniqueness theorem for the inverse problem.

Theorem 6.1. If $A_{m l}^{(1)}=A_{m l}^{(2)}$, for all $1 \leq l \leq M$, all $\alpha, \alpha^{\prime} \in S^{1}$, then $D_{1}=D_{2}$.

Proof. The basic idea of the proof is exactly the same as in Ref. 10. Using (6.1) and the assumption of the theorem, the difference of the scattering solutions $w:=u^{(1)}-u^{(2)}$ is $o\left(r^{-1 / 2}\right)$ outside of $D_{12}:=D_{1} \cup D_{2}$, and according to Theorem 1.1, $w$ vanishes in $D_{12}^{\prime}:=L \backslash D_{12}$. Therefore, in any connected component $\widetilde{D}$ of $D_{12} \backslash \overline{D^{12}}, D^{12}:=D_{1} \cap D_{2}$, and the bar denotes the closure, one has $u^{(1)}=u^{(2)}$ in $\widetilde{D}$, and, denoting by $v:=u^{(1)}=u^{(2)}$, one obtains $v=0$ on $\partial \widetilde{D}$. If $\widetilde{D}$ is not 
empty one gets a contradiction. The Dirichlet Laplacian in a compact region $\widetilde{D}$ has discrete spectrum, and in particular, the dimension of the eigenspace for any fixed eigenvalue is finite. On the other hand, choosing various $\alpha$ one can get as large dimension of the eigenspace corresponding to eigenvalue $k^{2}$ of the Dirichlet Laplacian in $\widetilde{D}$ as one wishes (see, e.g. Ref. 10, p. 88). Hence $\widetilde{D}$ must be empty, $D_{1}$ must coincide with $D_{2}$, and Theorem 6.1 is proved for the Dirichlet boundary condition on $S$.

Remarks: 1) A proof of the uniqueness Theorem 6.1 similar to that in Ref. 12 (see also Ref. 11) is also valid.

2) If the Neumann condition on $S$ is assumed, then the argument in the proof of Theorem 6.1 is not valid, because the Neumann Laplacian in domains with some non-smooth boundaries (the domain $\tilde{D}$ in the proof of Theorem 6.1, for example) may have non-discrete spectrum. However, in this case (provided that a uniqueness theorem similar to Theorem 2.1 is established) the argument in Ramm ${ }^{17}$ comes to rescue.

In Ref. 17 a new idea is introduced for the proof of the uniqueness of the solution to inverse obstacle scattering problem (IOSP). In order to explain the significance of this idea, let us recall that for some years there were assertions (both published and unpublished) that the method of M.Schiffer for proving uniqueness of the solution to IOSP breaks down in the case of Neumann boundary condition because the spectrum of the Neumann Laplacian in $\tilde{D}$ is not necessarily discrete (unlike the spectrum of the Dirichlet Laplacian) even in the case when the boundaries of the domains $D_{1}$ and $D_{2}$ are infinitely smooth. This observation was considered as one that invalidates the Schiffer's beautiful argument in the case of Neumann boundary condition. One way out of this difficulty is given in Ref. 13. The other way is given in Ref. 17. The idea in Ref. 17 is to avoid the reference to the discreteness of the spectrum of the Neumann (or the Dirichlet) Laplacian altogether, and to use instead the fact that the Hilbert space $L^{2}(\tilde{D})$ is separable, and therefore can have at most countable orthonormal basis. It is proved in Ref. 17 that if $D_{1}$ and $D_{2}$ are bounded Lipschitz domains which produce the same scattering data for $k$ in an open interval belonging to the positive semiaxis, and for all directions of the scattered wave, and if $\tilde{D}$ contains an open subset, then the Neumann (and the Dirichlet) Laplacian in $\tilde{D}$ has continuum of orthonormal in $L^{2}(\tilde{D})$ eigenfunctions, which is impossible since this space is separable. Thus, uniqueness of the solution to IOSP is established for Lipschitz obstacles and for both Neumann and Dirichlet boundary conditions, in domains, in which a uniqueness theorem is valid, which allows one to recover the scattered field outside the obstacle from the knowledge of the asymptotics of this field. In particular, this is the case for the domains considered in the present paper.

7. Existence of imbedded eigenvalues on the continuous spectrum of the Dirichlet Laplacian in some compactly perturbed waveguides

In this section we sketch the proof of the existence of imbedded eigenvalues 
of the Dirichlet Laplacian in a compactly perturbed waveguide provided that the perturbed waveguide has rotationally symmetric crossection and the crossection of the perturbed part of the waveguide is larger than the crossection of the original waveguide. This result is similar to the one by Witsch ${ }^{26}$ and was obtained as a partial answer to the question posed in Ref. 10, p.395, Problem 6. In Ref. ${ }^{26}$ the result is given for a compact perturbation of a half-cylinder, but the idea of our proof is the same.

Let us assume that the perturbed waveguide $W$ has the $z:=x_{3}$-axis as the axis of symmetry, that for $|z|>b$ the crossection of the waveguide does not depend on $z$ and is a circle of radius 1 , while there is an interval $a_{1}<z<a_{2}$ of the $z$-axis where the radius of the crossection is larger than 1 . Under the above assumptions, we claim that:

There are infinitely many positive eigenvalues of the Dirichlet Laplacian $A$ in $W$.

Let us prove this claim. Note that by symmetry an eigenfunction of $A$ in a perturbed cylinder $W_{m} \subset W$, formed by the surface of $W$ and the planes $\phi=0$ and $\phi=\pi / m$ (where $m$ is a positive integer which can be chosen as large as one wishes, and $\phi$ is the polar angle in the crossection of $W$ ), extends to an eigenfunction of $A$ in $W$ with the same eigenvalue by reflections in the planes $\phi=\ell \pi / m, \ell=0,1,2, \ldots, 2 m$, as one can check. By reflection, for example, in the plane $\phi=0$, we mean the transformation $u(r, \phi, z)=-u(r,-\phi, z)$, which allows one to check that the reflected function satisfies the Helmholtz equation in $\tilde{W}$ if the function $u$ satisfied it in the $W_{m}$. Here $\tilde{W}$ is the reflected region which is the union of $W_{m}$ and its reflection.

Let $W_{s} \subset W_{m}$ be a cylinder with height $d$ and the crossection which coincide with one of the crossections of $W_{m}$ with radius $r>1$. The Dirichlet Laplacian in $W_{s}$ has discrete spectrum and its first eigenvalue $\Lambda$ is arbitrary large if $m$ is sufficiently large. It is easy to see that $\Lambda>O\left(m^{2}\right)$ as $m$ grows. Since $W_{s} \subset W_{m}$, the variational principle for the eigenvalues implies that $A$ in $W_{m}$ has eigenvalues below the lowest point of its essential spectrum which are smaller than those of $A$ in $W_{s}$. The eigenvalues of $A$ in $W_{s}$ one can calculate by the separation of variables. They are equal to $\nu_{l}+\mu_{j}$, where $\nu_{l}$ are the eigenvalues of the Laplacian in the crossection of $W_{s}$ and $\mu_{j}$ are the eigenvalues of the operator $\frac{d^{2}}{d z^{2}}$ on the interval $[0, d]$, (where $d$ is the height of the cylinder $W_{s}$ ), with the zero boundary conditions at the ends. Note that $\nu_{l}=r^{-2} p_{l}$, where $p_{l}$ are the eigenvalues of $A$ in the crossection $W_{1}$ similar to the crossection of $W_{s}$ but with radius 1 . Let $l=1$ and we drop the index $l$ in what follows. Since $r>1$, one can get as many eigenvalues $\nu+\mu_{j}<p$ as one wishes, by choosing $m$ sufficiently large. All these eigenvalues lie below the essential spectrum of $A$ in the region $W_{1} \times(-\infty,+\infty)$, that is, below $p$. Let $R>r$, $W_{R}$ be the crossection similar to $W_{1}$ and let $T_{R}:=W_{R} \times(-\infty,+\infty)$. We assume that $W_{m} \subset T_{R}$. By the variational principle the eigenvalues of $A$ below its essential spectrum in $W_{m}$, that is, below $p$, are greater than the lowest point of spectrum of $A$ in $W_{R}$, equal to $\frac{p}{R^{2}}$ since $W_{m} \subset W_{R}$. Thus, we have proved that there are eigenvalues of $A$ in $W_{m}$ on the interval $\left(\frac{p}{R^{2}}, p\right)$, where $p$ can be chosen as large as one 
wishes by taking $m$ sufficiently large. But, as we explained above, each eigenvalue of $A$ in $W_{m}$ is an eigenvalue of $A$ in $W$ (with the reflected eigenfunction). Thus, the claim is proved.

\section{Remark on recent papers by R.Kleinman, T.Angell and coauthors}

After this work was finished and presented on June 4, 1997, at the ISAAC Congress, held at the University of Delaware, AGR learned of the two recent papers devoted to the same subject and available on the internet www.udel.edu

In this section comments are given on these papers which are reports of the center for the mathematics of waves at the University of Delaware, CW-1996-4 and CW-1996-11, supported by AFOSR grant F9620-96-1-0039, CNRS-NSF grant INT-9415493, and NATO grant CR6-940999.

These are papers CW-1996-4 (CW4), by Angell, Kleinman, Lesselier and Rozier, Uniqueness and complete families for an acoustic waveguide problem, and CW-199611, by Kleinman and Angell, Reciprocity, radiation conditions and uniqueness.

In CW4 an attempt is made to prove uniqueness theorem for the scattering problem for an acoustically hard obstacle placed in two-dimensional waveguide with boundaries which are two parallel straight lines.

Note that in Refs. 2 and 3 one can find counterexamples to such a uniqueness theorem, the proof of which in CW4 is erroneous: in particular, inequality (2.37) is obviously false since $K^{2}$ can be taken arbitrary large and $u(x)$ is a fixed function independent of $K$ and not vanishing identically.

Theorem 2.2 in CW4 asserts that "the only solution...which satisfy conditions of Theorem 2.1 is $u \equiv 0$ ", while in fact $u \equiv 0$ does not satisfy condition (2.7) of Theorem 2.1 since this condition is stated in CW4 with the strict inequality sign. We discuss below condition (2.7) in the form (8.1).

The main point in CW4 and CW11 is the introduction of the "new set of conditions..." in place of the radiation condition and its variants (see Ref. 10 where the role of the radiation condition is explained and its variants are discussed). The basic "new condition" is condition (2.7) in CW4, (which we give with the non-strict inequality sign by the reason explained in the preceding paragraph):

$$
\lim _{r \rightarrow \infty}\left[\Im \int_{|x|=r} \bar{u} u_{N} d s\right]=c=\text { const } \geq 0,
$$

where the unit normal $N$ is pointing outside the ball $|x|<r$, and condition (8.1) "should hold for a solution $u \neq \equiv 0$ to Helmholtz equation and any of its orthogonal decomposition components, that is, for any $u_{j}, j=1,2$, where $u=u_{1}+u_{2}$ and $\int_{|x|=r} u_{1} \overline{u_{2}} d s=0 . "$

We want to show that the "new condition" (8.1) is void, it is never satisfied: one can always find an orthogonal decomposition of $u$ for which $u_{1}$ does not satisfy (8.1). For simplicity, let us assume that the obstacle is placed in the whole space, this case is also included in the considerations of CW4 and CW11. 
Choose any function $f(x)=h(x)+i g(x)$, where $h$ and $g$ are real-valued, such that:

a) $f$ does not satisfy condition (1), and, in particular, does not vanish identically on the surface $|x|=r$,

and

b) $\int_{|x|=r} f \bar{u} d s \neq 0$.

There are infinitely many such functions.

Define $u_{1}=c f, u_{2}=u-u_{1}$, where $c=$ const is chosen so that $\int_{|x|=r} u_{1} \overline{u_{2}} d s=0$. This yields the formula for $c$ :

$$
|c|=\frac{\int_{|x|=r} f \bar{u} d s}{\int_{|x|=r}|f|^{2} d s}, \quad \arg c=-\arg \left[\int_{|x|=r} f \bar{u} d s\right] .
$$

The constructed $u_{1}$ does not satisfy condition (8.1): if $f$ does not satisfy (8.1) then clearly $c f$ does not satisfy it for any constant $c \neq 0$.

We have proved that the "new condition" from CW4 and CW11 is never satisfied.

To construct examples of $f$ which do not satisfy (8.1), one notes that the expression on the left-hand side in (8.1), in which $f=h+i g$ is substituted in place of $u$, is

$$
\lim _{|x| \rightarrow \infty} \int_{|x|=r}\left(h g_{N}-g h_{N}\right) d s=\int_{\mathbb{R}^{3}}(h \Delta g-g \Delta h) d x,
$$

where we assume that $h, g \in C^{2}$, and the integral on the right-hand side converges.

Take, for instance, $h=1$ and $\Delta g=G$, where $G$ is an arbitrary $L_{0}^{2}\left(\mathbb{R}^{3}\right)$ function such that $\int_{\mathbb{R}^{3}} h \Delta g d x=\int_{\mathbb{R}^{3}} G d x<0$, then condition (8.1) is not satisfied by $u=f$. There are infinitely many such $f$, and one can find among them many satisfying the condition $\int_{|x|=r} f \bar{u} d s \neq 0$, and such that condition (8.1) is not satisfied.

A specific example of $f$ in $\mathbb{R}^{3}$ is $f:=F:=r^{2}-i c r^{4}, c=$ const $>0$, for which the expression under the sign of limit in (8.1) is negative and tends to $-\infty$ as $r=|x|$ grows. If $\int_{|x|=r} \bar{u} d s \neq 0$, then one can choose $c>0$ sufficiently large so that $\int_{|x|=r} F \bar{u} d s \neq 0$, and then $F$ is the desired specific example. If $\int_{|x|=r} \bar{u} d s=0$, then, since $u(x)$ does not vanish identically, there is a real-valued normalized in $L^{2}\left(S^{2}\right)$ spherical harmonic $Y_{\ell}$ such that $\int_{|x|=r} Y_{\ell} \bar{u} d s \neq 0$. Let $f:=Y_{\ell} F$. Then this $f$ is the desired specific example.

The authors of CW4 write that "uniqueness in a waveguide was established by Sveshnikov by an application of limiting absorption principle" and refer to the paper by Sveshnikov A.G., Doklady Acad. Nauk USSR, 80, (1951), 341-344. In fact, the paper by Sveshnikov which deals with the waveguide whose boundaries are planes, is the paper Sveshnikov A.G., Doklady Acad. Nauk USSR, 73, (1950), 917-920. In this paper it is incorrectly claimed that the limiting absorption principle in the absence of an obstacle holds for the Helmholtz equation in $L$ for all $k>0$. In fact, this principle does not hold for $k \in \mathcal{K}$, in two- and three-dimensional spaces, as mentioned in Ref. 24, p.20. One can see this from the explicit formula for the resolvent kernel of the Dirichlet Laplacian in $L$, for example. 
In CW4 reference is made to [27], where, according to the authors of CW4, for the first time the uniqueness theorem for the boundary-value problem for soft obstacle in a waveguide was established. In fact, the proof of the uniqueness theorem in [27] is wrong. The correct proof of such a uniqueness theorem appears earlier in [8]. The author of [27] in his Ph.D thesis used the correct proof from [8]. 


\section{References}

1. D. S. Ahluwalia and J. B. Keller, Exact and asymptotic representation of the sound field in a stratified ocean, in Wave Propagation and Underwater Acoustics, eds. J.B. Keller and J.S. Papadakis (Springer-Verlag, 1977), pp. 14-85.

2. M. Callan, C.M. Linton and D.V. Evans, Trapped modes in two- dimensional waveguides, J. Fluid Mech. 229(1991) 51-64. a)D.V.Evans, M. Levitin and D.Vassiliev, Existence theorems for trapped modes, ibid., 261 (1994) 21-31.

3. D.V. Evans, C.M. Linton and F. Ursell, Trapped mode frequencies embedded in the continuous spectrum, Q. J. Mech. appl. Math.46(2)(1993) 253-274.

4. C.I. Goldstein, Eigenfunction expansion associated with the Laplacian for certain domains with infinite boundaries I, Trans. Amer. Math. Soc. 135 (1969) 1-31.

5. R.P. Gilbert and Y. Xu, Starting fields and far fields in ocean acoustics, Wave Motion 11 (1989) 507-54.

6. R.P. Gilbert and Y. Xu, Dense sets and the projection theorem for acoustic waves in a homogeneous finite depth ocean, Math. Meth. in the Appl. Sci. 12 (1989) 67-76.

7. R.P. Gilbert and Y. Xu, The propagation problem and far-field patterns in a stratified finite-depth ocean, Math. Meth. in the Appl. Sci. 12 (1990) 199-208.

8. K. Morgenröther and P. Werner, Resonances and standing waves, Math. Meth. in the Appl. Sci. 9 (1987) 105-126.

9. K. Morgenröther and P. Werner, On the principles of limiting absorption and limiting amplitude for a class of locally perturbed waveguides. Part 1: Time-independent theory, Math. Meth. in the Appl. Sci. 10 (1988) 125-144.

10. A.G. Ramm, Scattering by obstacles (Reidel, 1986).

11. A.G. Ramm, Multidimensional inverse scattering: solved and unsolved problems, Proc. Intern. Conf. on Dynamical Syst. and Applic., eds. G. Ladde and M. Sabandham (Atlanta, Georgia, 1994), pp.287-296.

12. A.G. Ramm, Scattering amplitude as a function of the obstacle, Appl. Math. Lett. 6 (5) (1993) 85-87.

13. A.G. Ramm, New method for proving uniqueness theorems for obstacle inverse scattering problem, Appl. Math. Lett. 6 (6) (1993) 89-92.

14. A.G. Ramm, The scattering problem analyzed by means of an integral equation of the first kind, J. Math. Anal. Appl. 201 (1996) 324-327.

15. A.G. Ramm and A. Ruiz, Existence and uniqueness of scattering solutions in nonsmooth domains, J. Math. Anal. Appl. 201 (1996) 329-338.

16. A.G. Ramm, Investigation of the scattering problem in some domains with infinite boundaries, I, II, Vestnik 7(19) (1963) 45-66, 67-76.

17. A.G. Ramm, Uniqueness theorems for inverse obstacle scattering problems in Lipschitz domains, Applic. Analysis 59 (1995) 377-383.

18. A.G. Ramm, Continuous dependence of the scattering amplitude on the surface of an obstacle, Math. Methods in the Appl. Sci. 18 (1995) 121-126.

19. A.G. Ramm, Stability of the solution to inverse obstacle scattering problem, J.Inverse and Ill-Posed Problems 2(3) (1994) 269-275.

20. A.G. Ramm, Stability estimates for obstacle scattering, J. Math. Anal. Appl. 188(3) (1994) 743-751.

21. A.G. Ramm, Examples of nonuniqueness for an inverse problems of geophysics, Appl. Math. Lett. 8(4) (1995) 87-90.

22. A.G. Ramm, Stability of the solution to $3 D$ inverse scattering problems with fixed-energy data, Inverse Problems in Mechanics, (Proc. ASME, AMD-Vol. 186 1994), pp. 99-102. 
23. A.G. Ramm, Remark on recent papers by R.Kleinman, T.Angell and coauthors,

24. A.G.Ramm and P.Werner, On limit amplitude principle, Jour. fuer die reine und angew. Math. 360 (1985) 19-46.

25. F. Rellich, Das Eigenwertproblem von $\Delta u+\lambda u=0$ in unendlichen Gebieten, Jber. $d$. deutsch. Math.-Verein. 53 (1943) 47-65.

26. K.J. Witsch, Examples of embedded eigenvalues for the Dirichlet- Laplacian in domains with infinite boundaries, Math. Meth. in the Appl. Sci. 12 (1990) 177-182.

27. Y. Xu, The propagating solution and far field patterns for acoustic harmonic waves in a finite depth ocean, Appl. Anal. 35 (1990) 129-151.

28. Y. Xu, An injective far-field pattern operator and inverse scattering problem in a finite depth ocean, Proc. Edinburgh Math. Soc. 43 (1991) 295-311. 